\title{
Achieving Robustness and Eco-Efficiency in Sustainability for A Distribution Network
}

\author{
Yenming J. Chen \\ Logistics Management, National Kaohsiung 1st Univ. of Sci \& Tech, No. 2 Jhuoyue Rd., Nanzih, Kaohsiung 811, Taiwan R.O.C
}

\begin{abstract}
This study investigates managerial insights to facilitate the robust construction of a responsive and sustainable supply chain network. The two goals typically contradict each other because a fast, responsive supply chain may consume sizable resources. A fully optimized supply chain, which operates at minimal cost, may not honor the two indicators simultaneously. Through a mathematical programming model assessing the necessary cost of a supply chain, this study argues that such a dilemma can be reconciled by reallocating stocking items across warehouse locations. Instead of designing an ultimate network to optimize responsiveness and eco-efficiency, the model merely serves as a vehicle for estimating the cost of implementing a nominal supply chain. In the process, the interaction between two performance indicators is clarified. The distribution responsiveness and eco-efficiency indicators comprise stock distribution, transportation link diversity, service level, and life cycle assessment. Understanding the relation between these two indicators helps to determine a cost-effective configuration which achieves eco-efficiency without sacrificing responsiveness.
\end{abstract}

Keywords-location-inventory network; network responsiveness; eco-efficiency; performance robustness

\section{INTRODUCTION}

The global eco-movement trend calls for increased environmental consideration when designing supply chains. A substantial amount of capital may be required to achieve significant improvement, and various performance indicators can be used to assess the capability of a supply chain. However, an increase in capital investment may not induce a commensurate increase in performances such as network efficiency or eco-efficiency. This study investigates the influence of distribution responsiveness and eco-efficiency on cost efficiency, which mainly refers to the selection of distribution center (DC) locations.

In adopting the concept of eco-efficiency and the environmental management system stated in ISO 14001, for example, reporting on the carbon dioxide (CO_2) emissions within the judicial borders of a company is insufficient. Furthermore, upstream suppliers, such as IBM, HP, and Foxconn, have pursued initiatives for environmental innovation. In general, companies must recognize their extended responsibilities and manage the entire life-cycles of their respective systems.

Responsiveness and operating costs do not always commensurate with each other. A DC tends to significantly reduce its inventory in each phase of the commodity circulation process for fast delivery because the cost and risk of goods on hand increase. Customers typically request the instant replenishment of supplies, and a short lead time for order fulfillment is preferred because customers cannot tolerate shortage. To meet the requirement of retail terminals for replenishment, DCs must be capable of delivering quickly. Effective supply chain management can enhance the speed and elasticity of enterprises, as well as reduce operating cost while improving the capacity to promise delivery to customers. Conventional management strategies seek to address the uncertain requirements of customers by constructing DCs or enhancing prepared inventories. Such strategies increase facility and inventory costs. Unfortunately, increasing operating costs does not necessarily translate to a tangible improvement in performance because the relation between cost and performance is nonlinear stepwise.

[10] develop an integrated production and inventory model that comprises factories, transportation, and the cost of safety stocks. [12] extend the location-inventory model to consider supply uncertainty in reverse logistics. [8] integrate lead time into a responsive supply chain as a cost-effective strategy for linking production and logistics. [5] argues that inventory item reallocation can enhance network efficiency and network responsiveness by at least $10 \%$. However, the supply chain networks investigated by these models do not consider the robustness issue for item assignment in the network.

[8] quantize supply chain responsiveness by considering inventory under the condition of uncertain demand. [3] gain the network responsiveness for an optimal multi-phase supply chain in consideration of product sales, delivery, production shortage, delivery delay, storage, and work orders. Aside from efficiency, responsiveness generally implies more cost. [7] believe that the response speed of a supply chain can be improved by expanding operational facilities through additional investments. Many studies have also proposed methodologies for measuring responsiveness. [1] argue that rapid responsiveness must exhibit six traits, namely, order fill rate, order fulfillment reliability, new product introduction, time to market, production lead time and customer response capability.

When institutional pressures increase, companies must effectively manage sustainability for their supply chain [13]. The current research is the first to consider responsiveness and eco-efficiency for an operational network. We investigate how a properly designed logistics network can improve responsibility while maintaining necessary operating cost. We also determine the point at which eco-efficiency enhancement coincides with the goal to improve network efficiency and how the aforementioned network can help meet environmental goal. In this study, the ideal location for candidate DCs and the 
optimal item inventory for all distributors are determined on the basis of the optimal item-reallocation. We then analyze the responsiveness and eco-efficiency of the suggested distribution network. Two solutions are considered to achieve responsiveness and eco-efficiency. We can sacrifice a small amount of cost efficiency in exchange for incorporating these two performance indicators. We can also reassign stocking items to the optimal locations to retain performance with comparative minimal cost. We find that the item reallocation method is robust in achieving the two performance goals.

\section{MODEL}

The distribution network presented in this study consists of three echelons: the supplier, DCs, and retail customers. The stocking quantity for each DC should be considered in the distribution network. We assume that the quantity provided by the supplier is known and fixed. The index $l \in D$ pertains to the candidate DCs; $i \in C$ refers to retail customers; and $k \in K$ represents the items that pass through the distribution network. The binary decisions $x_{j}$ and $y_{i}$ indicate whether or not depot $j$ is selected as a DC and serves customer ${ }^{i}$, respectively. Each retailer ${ }^{i}$ requests an average demand $u_{i k}$ for item $k$ annually. A DC $j_{\text {with capacity }} c_{j}$ requires a unit construction cost $f_{j}$ to build and a unit operating cost $w_{j}$ to operate. To maintain the basic functionality of a distribution network, each DC must retain a working inventory $s_{j k}$ and a minimal level of safety stock that depends on the mean $\mu_{i}$ and variance $\sigma_{i}^{2}$ of demand. In addition, $z_{\alpha}$ represents the level at which $\operatorname{Pr}\left(z \leq z_{\alpha}\right)=\alpha$. Let $h$ denote the inventory holding cost per unit of a product per year. The delivery cost $d_{i j}$ consists only of the transportation cost from DC $j$ to retail outlets $i$.

The program can be expressed as

$\min$

$$
\begin{aligned}
& \sum_{j \in D, k \in R}\left\{f_{j} c_{i} x_{t}+\sum_{i \in C} d_{i j} y_{i j}+w_{j} s_{j k}+h z_{\alpha x} \sqrt{\sum_{i \in C} \sigma_{i}^{2} y_{i t}}\right\} \\
& \text { s.t. } \sum_{t \in D} x_{t}=1 \text {, }
\end{aligned}
$$

$$
\begin{aligned}
& y_{i k k}-x_{i} \leq 0, \forall i \in D_{n} j \in C_{n} k \in K_{u} \\
& \sum_{j \in D} s_{j k}=\sum_{i \in C} \tau_{i k}, \forall k \in K_{n} \\
& c_{i} x_{j} \geq s_{j k} \geq \sum_{i \in C} u_{i k} y_{i j k}, \forall j \in D, k \in K, \\
& x_{i p} y_{i j k} \in\{0,1\}, \forall t_{n} j .
\end{aligned}
$$

Objective (1) consists of four parts, namely, the total fixed costs of DCs, total transportation cost, total operating cost, and total safety stock cost. Constraint (2) indicates that at least one DC must be selected from all candidates. Constraint (3) implies that the retailers can only be replenished by a working DC. Constraint (4) suggests that total inventory and total demand should be balanced. Constraint (5) ensures that the allocated inventory in each DC is sufficient to fulfill retailer requirements and the allocation should be zero if the candidate DC is not selected.

Order fill rate and continuous supply rate should be regarded as key factors in enhancing responsiveness [1]. Maneuverability consists of three factors, namely, location redundancy, supply multiplicity, and delivery versatility. These factors correspond to three quantification components, that is, the levels of inventory decentralization, inventory dispersion, and transportation diversity.

The quantification of each indicator component is in different scales. Therefore, we must rescale them before we can add these components together. The components in question are detailed as follows.

- Level of inventory decentralization: Inventory decentralization refers to the scattering of geographic locations of distribution network facilities. This factor significantly affects maneuverability. Centralizing inventories in a few locations can achieve the efficiency of a scale economy, whereas decentralizing inventories in multiple locations that are near customers and suppliers can improve responsiveness to the timely demands of customers. We use the term $\left(\sum_{i=R 2}\left(\sum_{i \in C_{i}, k=R} y_{i j k}\right)^{2}\right)^{-c 12}$ to decentralization or location redundancy. Strategic decisions related to inventory locations are crucial because such choices affect many long-term plans and involve a significant amount of funds. A company can emphasize its responsiveness by establishing several operating facilities in regions near its target customers. For example, Bridgestone Tires set up many warehouses in high-scale locations to improve responses to customer demands. A major advantage of a decentralized network is on system robustness. If one location fails, then its customers can be served by adjacent or high-level locations. Thus, location redundancy can strongly promote responsiveness.

- Level of inventory dispersion: The amount of inventory directly influences responsiveness [2]. Inventories are distributed throughout the entire supply chain, from raw materials and products in process to end products. Thus, managers must assess the conflict between maneuverability and operating cost. Inventory decisions made by the distribution network primarily focus on working inventory and safety stock. Storing inventories in multiple locations can allow companies or overall supply chains to respond rapidly in fluctuating customer demands. However, the multiplicity of inventory holding causes the swift accumulation of inventories and generates a serious liquidity problem. We employ the term $\left(\sum_{\in \in C, k \in R}\left(s_{i k}\right)^{2}\right)^{-\frac{1}{2}}$ to reflect the multiplicity of supply in multiple locations. To maintain the operating efficiency, the accumulated amount of inventories cannot increase without limit.

- Level of transportation diversity: As a key factor in a distribution network, transportation accounts for more than 
$30 \%$ of logistic cost. Thus, the proper management of transportation not only affects logistic cost but customer satisfaction as well. We exploit the term $\left(\sum_{i \in C_{j} \in R}\left(\sum_{k \in k}, v_{v j k} d_{v t}\right)^{2}\right)^{-\frac{1}{2}}$ to represent the delivery versatility in network maneuverability. In contrast to inventory decentralization, which reduces transportation time and induces a rapid response to customer demands, transportation diversity increases responsiveness by adding parallel routes to overall maneuverability. Centralizing delivery in a large-scale DC may yield economies of scale but also reduce the number of accessible links from one location to another.

- Level of resource benignity: Unlike transportation, resource benignity can benefit both responsiveness and ecoefficiency simultaneously. The quick response of a supply chain is commonly associated with resource consumption. In this study, resource benignity refers to the reciprocal of LCA [25], and we use the term $\left(\sum_{i \in C, j \in \mathbb{D}, k \in R} y_{t j k}\right)^{-1}$ to represent the tendency for small values to imply the effective use of resources.

\section{COMPROMISED SOLUTION TO ACHIEVE ROBUSTNESS}
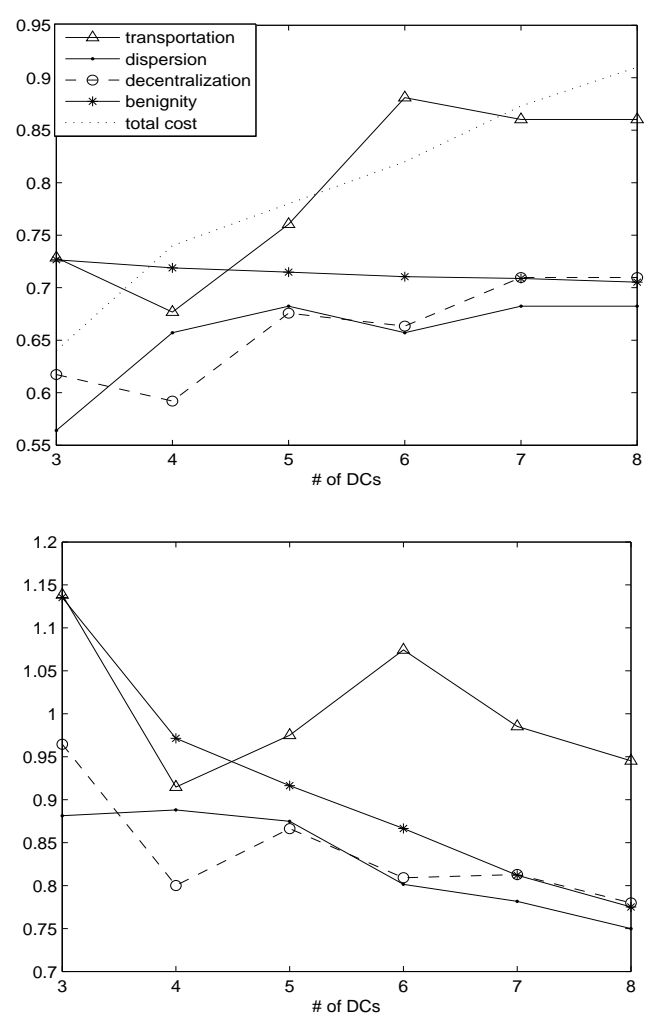

FIGURE I. COMPROMISE OPTIMALITY FOR ROBUSTNESS. (A) INDIVIDUAL VALUES; (B) VALUE TO COST RATIOS

To retain the robustness of network performance, a planner often compromises the optimized solution and selects a less optimized solution to achieving stable responsiveness and ecoefficiency. As shown in Fig. 1, the optimal number of DCs is 4 for the basic configuration. Three is the optimal number when capacity constraints are ignored. We then deliberately increase the number of DCs from 4 to 8, and the results exhibit a stepwise increase in total cost. Subgraph (a) of Fig. 1 shows the changes in the index components when the number of operating DCs is increased. Following the process of value normalization, transportation capability increases significantly, as opposed to the rise in inventory dispersion and network decentralization. This phenomenon is particularly clear when the number of operating DCs varies between 4 and 6 . As expected, resource benignity decreases when the requirement for resources increases. However, network responsiveness increases in subgraph (b) because the levels of both transportation and decentralization increase. The effect of the compromise in configuration has indefinitely influences ecoefficiency. If the weight of energy conservation is greater than that of resource conservation, then eco-efficiency is improved by the compromise solution

\section{CONCLUSIONS}

This study analyzes the responsiveness and eco-efficiency of distribution networks when enterprises plan for the longterm. Implementation cost should be constantly minimized to facilitate the network efficiency of a supply chain. We also investigate two solutions to achieve responsiveness and ecoefficiency. The first approach involves compromising system cost by selecting the second optimal solutions. The second method involves reassigning stocking items to optimal locations. The results indicate that the item reallocation method is robust for choosing versatile network configurations. Future studies should incorporate the uncertain factors of demand and consider the effect of scale economies in transportation.

\section{REFERENCES}

[1] Agarwal, A., Shankar, R., Tiwari, M., 2006. Modeling the metrics of lean, agile and leagile supply chain: An anp-based approach. European Journal of Operational Research 173 (1), 211-225.

[2] Blumenfeld, D. E., Daganzo, C. F., Frick, M. C., Gonsalvez, D. J., 1999. Impact of manufacturing response time on retailer inventory. International Journal of Operations \& Production Management 19 (8), 797-811.

[3] Bok, J.-K., Grossmann, I. E., Park, S., 2000. Supply chain optimization in continuous flexible process networks. Industrial \& Engineering Chemistry Research 39 (5), 1279-1290.

[4] Chen, Y. J., 2011. Regional diffusion for remanufactured tied products and ecologically conscious consumers. International Journal of Sustainable Economy 3 (4), 440-454.

[5] Chen, Y. J., 2014. Item-location assignment to responsiveness for fulfilling rush demands in a manufacturing network. International Journal of Modeling and Optimization 4 (6), 493-498.

[6] Chen, Y. J., Sheu, J.-B., 2009. Environmental-regulation pricing strategies for green supply chain. Transportation Research Part E: Logistics and Transportation Review 45 (5), 667-677.

[7] Chopra, S., Meindl, P., 2007. Supply chain management. Strategy, planning \& operation. Springer.

[8] Christopher, M., Towill, D., 2001. An integrated model for the design of agile supply chains. International Journal of Physical Distribution \& Logistics Management 31 (4), 235-246.

[9] Czaplicka-Kolarz, K., Burchart-Korol, D., Krawczyk, P., 2010. Ecoefficiency analysis methodology on the example of the chosen 
polyolefins production. Journal of Achievements in Materials and Manufacturing Engineering 43 (1), 469-475.

[10] Daskin, M., Coullard, C., Shen, Z., 2002. An inventory-location model: Formulation, solution algorithm and computational results. Annals of Operations Research 110 (1), 83-106.

[11] Ehrenfeld, J. R., 2005. Eco-efficiency. Journal of Industrial Ecology 9 (4), 6-8.

[12] Fleischmann, M., Beullens, P., Bloemhof-Ruwaard, J., Wassenhove, L. 2009. The impact of product recovery on logistics network design. Production and Operations Management 10 (2), 156-173.

[13] Hall, J., 2000. Environmental supply chain dynamics. Journal of Cleaner Production 8 (6), 455-471.

[14] Hart, S., 1997. Beyond greening: Strategies for a sustainable world. Harvard Business Review 75 (1), 66-76. 